\title{
LA RELACIÓN FAMILIA-INSTITUCIÓN EDUCATIVA EN ENSEÑANZA MEDIA: PERSPECTIVAS DE DOCENTES DE SECUNDARIA
}

\author{
The Relationship Between Family and School in High School Education. \\ The Perspective of High School Teachers
}

\author{
RODRIGO VACCOTTI*1 \\ http://orcid.org/0000-0002-9650-8997 \\ Universidad de la República. Uruguay. \\ Correspondencia: rodrigovaccottimartins@gmail.com
}

Recibido: 24-02- 2017

Revisado: 23-05-2017

Aceptado: 16-07-2017

\begin{abstract}
Resumen: Las dificultades asociadas a la relación familia-institución educativa aparecen cada vez en mayor medida como objeto de discusión en la bibliografía internacional. El presente estudio se propone indagar acerca de las perspectivas de los docentes de educación secundaria acerca de la relación familia-institución educativa. Para esto se contó con un diseño metodológico de corte cualitativo que incluyó dos focus group entre docentes de secundaria que, al momento del estudio, trabajaban en primer año de ciclo básico. Los datos generados fueron luego estudiados mediante la técnica de análisis de contenido. Entre los principales resultados de la investigación, que supone un primer acercamiento a la temática, se destaca que los docentes consideran fundamental la participación de las familias, pero esta no se da de modo adecuado debido a que las familias muestran muchas dificultades, las instituciones no tienen lineamientos claros para facilitar su acercamiento y la formación de los docentes en este rubro es deficitaria.
\end{abstract}

Palabras clave: relación familia-escuela; educación secundaria; docente; formación docente.

\begin{abstract}
:
Difficulties associated with relationship between family and school appear increasingly as a matter of discussion in the international bibliography. This study seeks to investigate the perspectives of secondary school teachers on the relationship between family and school. For this purpose, a qualitative methodological design was used, including two focus groups among secondary school teachers who, at the time of the study, worked in the first grade. The data generated were then studied using the content analysis technique. Among the main results of the research, which presupposes a first approach to the subject, it stands out that teachers consider the participation of families to be fundamental, but this is not done in an adequate manner because families show some difficulties, institutions do not have clear guidelines to facilitate their approach, and teacher training in this area is insufficient.
\end{abstract}

Keywords: family-school relationship; high school; teacher; teacher's training.

\footnotetext{
${ }^{1}$ Dr. (c) en Ciencias Sociales de la Universidad Nacional General Sarmiento (Argentina). Magíster en Psicología Educacional en la Universidad Católica del Uruguay. Licenciado en Psicología en la Universidad de la República (Uruguay). Líneas de trabajo: juventud, educación, subjetividades juveniles, instituciones educativas.
} 


\section{INTRODUCCIÓN}

La relación familia-institución educativa y las dificultades que presenta este vínculo vienen siendo objeto de discusión y esto es reportado de manera muy numerosa en la bibliografía internacional en las últimas tres décadas. Si bien se ha establecido desde varios enfoques y aportes la necesidad de generar acuerdos entre ambos sistemas, así como estrategias que permitan generar mejoras en los modos de inclusión, las respuestas adecuadas parecen aún no encontrarse y ambas, familia e institución educativa, dan la sensación de transitar por caminos separados, e incluso, opuestos.

Llama la atención también un fenómeno que tiene lugar en el pasaje de las instituciones de educación primaria hacia las de educación secundaria y es el descenso en la presencia de la familia, el cual está asociado a varias razones.

Es notorio, tanto en este punto como en la bibliografía y las bases de datos, que al referirse a este tema el hincapié principal está puesto en educación primaria, incluso utilizándose de modo natural para referirse al asunto el término la relación familiaescuela. Es por ello por lo que es necesario esclarecer qué es lo que ocurre con la participación de las familias en educación secundaria y por qué las dificultades en el vínculo parecen ser aún mayores que las existentes en primaria.

Otro de los puntos clave para este trabajo tiene que ver con el lugar que ocupan los docentes de secundaria y sus perspectivas, actores fundamentales a la hora de pensar esta problemática. Con respecto a estos genera especial interés el tema de su identidad, valoración social y su formación.

El estudio presentado aquí se encuentra asociado al trabajo realizado en el proyecto de investigación: La relación familia-escuela: perspectivas y formación de profesores brasileros y uruguayos (González Tornaría y Wagner, 2011), dentro del cual se trabajó en la temática durante el período de tiempo comprendido entre octubre de 2011 y octubre de 2013, generando insumos muy valiosos. En dicho proyecto se trabajó con focus group (FG) integrados por docentes de primaria, así como con padres uruguayos y brasileros, y a partir de los datos generados en esa instancia es que surge el interés de continuar profundizando en la temática, prestando especial atención a lo que acontece en educación secundaria.

\section{LA RELACIÓN FAMILIA-ESCUELA}

La dificultad que supone el establecer una definición de familia, más allá de ser un duro desafío, funciona para el investigador como información relevante a la hora de entender que sobre esta temática existe una diversidad muy grande de enfoques y concepciones.

El concepto de familia se ha ido modificando de acuerdo a las variables históricas, sociales y culturales. Rodrigo y Palacios (1998) se refieren a la familia en el mundo occidental y sobre fines del siglo XX de la siguiente manera: 
Se trata de la unión de personas que comparten un proyecto vital de existencia en común que se quiere duradero, en el que se generan fuertes sentimientos de pertenencia a dicho grupo, existe un compromiso personal entre sus miembros y se establecen intensas relaciones de intimidad, reciprocidad y dependencia (p. 33).

A su vez, establecen que no es excluyente que exista un matrimonio para hablar de familia; no necesariamente tienen que estar presentes los dos progenitores; los hijos son tenidos en común frecuentemente, pero esto no es condición necesaria; la madre no tiene la necesidad de dedicarse exclusivamente al cuidado de los hijos, así como no la tiene el padre de ser quien genera los recursos; el número de hijos se ha visto reducido; junto a separaciones y divorcios, algunos núcleos familiares se disuelven; es fundamental la interdependencia, comunicación e intimidad entre los adultos implicados; hay una relación de dependencia entren quien cuida y educa por sobre quien es cuidado y educado (Rodrigo y Palacios, 1998).

Además de presentar estas características, la familia cumple las siguientes funciones: Es dentro de la familia donde se construyen personas adultas, las cuales muestran una determinada autoestima, así como un bienestar psicológico directamente relacionado con la calidad de las relaciones de apego que estas personas han experimentado desde su niñez. Dentro de la familia los individuos aprenden a afrontar retos y a asumir las responsabilidades y compromisos que les impone el contexto en el que se desenvuelven. Allí se produce también un encuentro intergeneracional donde se establecen nexos entre el pasado y el futuro, entre la generación de los abuelos y la de los hijos. Por último, esta funciona como una red de apoyo social para las diferentes transiciones vitales que atraviesan los individuos. También es competencia de los adultos para con los más jóvenes asegurar su supervivencia, socialización, sano crecimiento, diálogo y simbolización; brindarles a estos un clima de afecto y apoyo necesario para un desarrollo psicológico sano; estimularlos para convertirse en seres con capacidad para relacionarse con el contexto que los rodea y cumplir con las demandas y exigencias que este plantea; y por último, tomar decisiones sobre el resto de los contextos educativos que transitarán los hijos (Rodrigo y Palacios, 1998).

Lo educativo entonces entra claramente dentro de lo que entendemos como las funciones de la familia. El hecho de que esta no sea vista como un espacio donde se producen aprendizajes es porque existen en el colectivo social creencias acerca de la educación formal, que es considerada como la única encargada de generar aprendizajes en los más jóvenes (González, Vandemeulebroecke y Colpin, 2001).

Minuchin y Fishman (1984) hacen referencia al carácter dinámico de la familia, tomando este concepto no solamente para la institución familia, sino también para los casos concretos: "La familia no es una unidad estática. Está en proceso de cambio continuo, lo mismo que sus contextos sociales" (p. 34).

Nos encontramos al día de hoy con nuevas configuraciones familiares que presentan características propias del momento histórico al que pertenecen, marcadas, 
entre otras características, por una "liberación" por parte de los individuos de ciertos roles establecidos, propios de la modernidad (Walsh, 2004; Paredes, 2003). En el seno de estas familias y sus complejidades es necesario detenerse en la tarea de ser padres. Esta está guiada en buena medida por teorías implícitas, constituidas por un saber mediado por la interacción de un individuo y su cultura, y las experiencias a las que han asistido en sus propias familias de origen, así como en sus trayectorias de vida. Pensando específicamente en el contexto que se viene describiendo y en las transformaciones que ha experimentado la familia como institución, esta tarea se complejiza aún más (Rodrigo, Máiquez, Martín y Byrne, 2008).

Si bien puede pensarse que familia e institución educativa son dos partes de un mismo proceso - educar a los niños y jóvenes - queda claro que existen diferentes obstáculos para que funcionen juntos, generándose por momentos hasta una ruptura entre ambos sistemas (Castro y Regattieri, 2012). La escuela como tal no existe desde siempre. Es luego de la revolución industrial que surge como una necesidad que los individuos sean educados fuera de su casa. (Lacasa, 1997; Palacios y Oliva, 2008). La obligatoriedad de la escolarización ejerce coercitivamente sobre los padres la obligación de cederle una gran parte de la responsabilidad de la educación de sus hijos a la institución educativa (Perrenoud, 2010).

Existe una relación directa entre el modo en que tienen lugar las relaciones adulto-joven dentro de la familia y el modo en que estas tienen lugar dentro de la institución escolar; los conflictos intergeneracionales que acompañan las transformaciones que ha experimentado la familia, sobre todo para con las figuras de autoridad, están estrechamente vinculados con el clima que se vive en las escuelas, por lo que el trabajo sobre esta temática incumbe a ambas instituciones y debería ser abordado en conjunto (Gallo, 2009).

En la relación familia-institución educativa intervienen variables legales. En Uruguay existe la Ley General de Educación N.ำ18.437 (2008), publicada el 16 de enero de 2019, que hace referencia a este tema en su artículo IX, acerca de los "Derechos y deberes de los educandos y de madres, padres o responsables", donde se afirma que será deber y obligación de los padres, madres o responsables el seguir y apoyar el proceso de aprendizaje de su hijo o representado.

Con respecto a esto Alfiz (1997) establece que no siempre que se lleve a cabo una actividad en la que estén presentes ambas partes _familia y escuela - se puede hablar de participación, así como tampoco se puede presuponer que esto vaya a generar mejoras en la calidad educativa.

Existen diferentes niveles posibles de participación de las familias en los procesos educativos de sus hijos, encontrándose desde el mayor nivel, constituido por aquellas decisiones fundamentales tomadas por la familia, hasta el menor, en donde se ejecutan decisiones tomadas por otros, o donde incluso se participa pasivamente (Alfiz, 1997). 


\section{ASPECTOS METODOLÓGICOS}

Los objetivos de la investigación fueron los siguientes:

1) Indagar acerca de las perspectivas de los docentes de secundaria sobre la importancia de la participación de la familia en la educación.

2) Indagar acerca de qué diferencias entienden los docentes que existen en la participación de la familia en la educación entre los sistemas de primaria y secundaria.

3) Conocer sobre la formación con la que cuentan los docentes de secundaria para el trabajo en la relación familia-institución educativa.

Las principales preguntas que se intentaron responder mediante la investigación fueron las siguientes:

¿Cuáles son las perspectivas de los docentes de secundaria acerca de la relación familia-institución educativa? ¿Consideran los docentes que es importante la participación de las familias en los procesos de aprendizaje de los alumnos en enseñanza secundaria? ¿Qué diferencias en la participación de la familia consideran los docentes que existen entre primaria y secundaria? ¿Cuáles son las perspectivas de los docentes acerca de su formación? ¿Cuentan los docentes de secundaria con formación para el trabajo con las familias? ¿Consideran los docentes que es importante estar formado para el trabajo con las familias?

La estrategia de investigación se basó en un estudio exploratorio, con el objetivo de dar cuenta de los objetivos señalados en la sección anterior. Partiendo de las preguntas de investigación y de dichos objetivos se tomó la decisión de optar por un diseño de investigación de tipo cualitativo exploratorio. Se optó por un diseño metodológico flexible, una articulación de elementos que permita introducir durante el transcurso de la investigación los cambios que sean necesarios para captar los aspectos relevantes de la realidad analizada (Maxwell, 1996).

Este diseño se basa en técnicas de investigación cualitativas, ya que el objetivo es producir datos empíricos y elementos teóricos que aporten a la interpretación y explicación de un objeto de estudio que se basa en gran medida en la diversidad de sus participantes y sus perspectivas, de acuerdo a la naturaleza del problema de investigación, donde las dimensiones lingüística y simbólica son aspectos centrales para la comprensión de la temática (Flick, 2004; Vasilachis de Gialdino, 2007).

Se trabajó en dos instancias de focus group (Pérez De León, 2001; Flick, 2004). El proceso de selección de los docentes participantes fue realizado mediante el modelo de muestreo teórico (Glaser y Strauss, 1967) con el propósito de lograr un conjunto heterogéneo de sujetos tomando en cuenta características que consideramos relevantes, como ser: que los participantes pertenezcan tanto al sistema público como al privado; que los participantes desarrollen sus tareas en diferentes contextos 
socioculturales; que las edades y años de trabajo de los participantes sean variadas; que las disciplinas que imparten los participantes sean variadas y que los participantes tengan diferentes experiencias en cuanto a la formación específica para el trabajo con familias. Los docentes con los que se realizó el estudio necesariamente debían trabajar y haber trabajado anteriormente con primer año de Ciclo Básico al momento de participar del grupo.

Para el análisis de los datos generados a partir de los focus group se trabajó con la técnica de análisis de contenido. Para ser analizados los datos brutos deben ser codificados, es decir, transformados para poder realizar dicho análisis. El discurso de los docentes fue codificado en su totalidad en temas: el tema es una unidad de significado que se desprende del texto analizado. El total de temas identificado fue agrupado en diferentes categorías: clasificar elementos en categorías, es buscar lo que éstos tienen en común y, en definitiva, organizarlos. Las reglas de enumeración de los temas a partir de las cuales se trabajó en este estudio son las siguientes (Bardin, 1986): presencia; frecuencia; frecuencia ponderada; intensidad; dirección; orden y contingencia.

Un conjunto de buenas categorías debe tener las siguientes cualidades, en las cuales se basó el trabajo realizado en este estudio (Bardin, 1986): exclusión mutua; homogeneidad; pertinencia; objetividad y fidelidad, y productividad.

El primer focus group estuvo conformado de la siguiente manera:

Tabla 1. Cuadro descriptivo docentes FG 1

\begin{tabular}{|l|l|l|l|l|l|l|}
\hline Docente & Edad & $\begin{array}{c}\text { Años de } \\
\text { experiencia }\end{array}$ & Asignatura & $\begin{array}{c}\text { Experiencia en } \\
\text { educación pública }\end{array}$ & $\begin{array}{c}\text { Experiencia en } \\
\text { educación privada }\end{array}$ & $\begin{array}{l}\text { Madrel } \\
\text { Padre }\end{array}$ \\
\hline S1 & 36 & 5 & Id. Español & Sí & Sí & Sí \\
\hline I1 & 42 & 5 & Id. Español & Sí & Sí & No \\
\hline P1 & 38 & 12 & Inglés & Sí & Sí & Sí \\
\hline C1 & 42 & 8 & Inglés & Sí & Sí & No \\
\hline R1 & 47 & 21 & Informática & Sí & Sí & Sí \\
\hline H1 & 38 & 13 & Matemática & Sí & Sí & Sí \\
\hline
\end{tabular}

Fuente: Elaboración propia (2014)

Todas las participantes son docentes egresadas del IPA que cursaron el Plan 1986. Todas las participantes del grupo son mujeres. 
El segundo focus group estuvo conformado de la siguiente manera:

Tabla 2. Cuadro descriptivo docentes FG 2

\begin{tabular}{|l|l|l|l|l|l|l|}
\hline Docente & Edad & $\begin{array}{c}\text { Años de } \\
\text { experiencia }\end{array}$ & Asignatura & $\begin{array}{c}\text { Experiencia en } \\
\text { educación pública }\end{array}$ & $\begin{array}{c}\text { Experiencia en } \\
\text { educación privada }\end{array}$ & $\begin{array}{c}\text { Madre/ } \\
\text { Padre }\end{array}$ \\
\hline N2 & 27 & 6 & Historia & Sí & Sí & No \\
\hline T2 & 31 & 3 & Literatura / ECA & Sí & Sí & No \\
\hline M2 & 36 & 10 & Cs. Físicas & Sí & Sí & Sí \\
\hline F2 & 28 & 8 & Música & Sí & Sí & No \\
\hline C2 & 28 & 5 & Id. Español & Sí & Sí & No \\
\hline
\end{tabular}

Fuente: Elaboración propia (2014)

Todos los participantes son docentes son egresados del IPA que cursaron el Plan 1986. Cuatro de las participantes son mujeres y el restante es un varón (N2).

Del primer focus group, los temas que aparecieron con mayor frecuencia e intensidad fueron aquellos relacionados con el rol docente, la formación, el manejo de la información y la falta de acompañamiento que presentan los adolescentes. Del segundo focus group, los temas que aparecieron con mayor frecuencia e intensidad fueron aquellos relacionados con el rol docente, y la formación.

\section{ANÁLISIS DE LOS DATOS}

En este trabajo se analizaron las perspectivas de los docentes de secundaria acerca de la relación familia-institución educativa en enseñanza media. Para indagar acerca de esto se utilizó la técnica focus group. Se trabajó en dos instancias y para esto se contó con la colaboración de 11 docentes de secundaria que trabajan actualmente en $1 .^{\text {er }}$ año de ciclo básico, en enseñanza pública y privada, así como en diferentes contextos socioculturales en Montevideo y en algunas ciudades del interior del país.

Mediante la interacción que se produjo entre los participantes en los focus group y la diversidad de miradas y opiniones que tuvo lugar allí se pudo generar un acercamiento a la temática, que contribuye un aporte importante para continuar trabajando la relación familia-institución educativa. Para la interpretación de los datos arrojados por la investigación se trabajó con la técnica Análisis de contenido (Bardin, 1986). 
Cada grupo fue trabajado por separado y recién sobre el final del estudio se establecieron algunas comparaciones entre los resultados que surgieron de ellos, comparaciones sobre las que se reflexionará a continuación, para luego pasar a la discusión y conclusiones generales.

En este trabajo, además de hacer hincapié en las perspectivas de los docentes acerca de la relación familia-institución educativa y la formación docente, se tomaron en cuenta las variables socioculturales propias de nuestro contexto.

\section{Comparación de los focus group}

Existe una diferencia entre los promedios de edad de ambos FG (40.5 > 30) así como entre los promedios de años de experiencia $(10,6>6.4)$. Las categorías en las que se organizaron ambos FG tienen bastantes similitudes, aunque el tipo de posicionamientos que surgen con respecto a los temas asociados al rol docente y a la formación muestran diferencias interesantes. Esto nos lleva a pensar, como una de las variables, en las diferentes etapas del desarrollo docente descritas por Day (2005). Los temas asociados al rol docente aparecen en ambos FG con gran frecuencia e intensidad y se hace hincapié en esta temática, aunque de modos distintos. En los siguientes parlamentos se pueden ver diferentes perspectivas de los docentes que nos muestran un determinado malestar relacionado a las tareas que estos deben cumplir, las cuales derivan en cuestionamientos acerca del rol docente.

FG 1:O sea, como lo que vos decías, yo en la clase quiero ir y enseñar idioma español, no quiero ir y hacer de psicóloga porque no lo soy, no quiero, no es para lo que estudié (S1).

FG 2: Para mí lo más patético, y que los gurises aparte te lo reclaman, o sea, no son idiotas ¿no? Es el tema de las preguntas diagnósticas... no la prueba diagnóstica, sino la parte social digamos... ¿qué música escuchás? Que no sé qué, que no sé cuánto... a nosotros por ejemplo en didáctica era como que, tenés que preguntar con quién vivís... y ¿qué haces con eso? Porque la pregunta siempre está (T2).

Aquí aparecen relatadas dos situaciones en las que los docentes se encuentran en desacuerdo con determinadas tareas que deben realizar. La diferencia radica en que mientras el docente del FG1 está realizando una crítica acerca del apoyo que le falta por parte de la institución para solucionar las situaciones conflictivas que se generan, el docente del FG2 es crítico para con los lineamientos de secundaria acerca de cómo debe llevar adelante su curso. En los siguientes fragmentos de los parlamentos de docentes de ambos grupos se refuerza esta idea. A través de situaciones distintas, el denominador común es que los docentes tienen dificultades para definir cuál es específicamente su rol, quedando claro sí que existe malestar con el modo en que lo están desarrollando actualmente. Una vez más, en el FG1 se pone el énfasis en 
situaciones que ocurren dentro la institución para las cuales no cuentan con apoyo, mientras que en el FG2 se hace hincapié en lo que ocurre dentro del salón de clase.

FG1: $Y$ además hay veces que te empezás a extender, y la familia hay veces que te empieza a decir, se empiezan a desahogar contigo y vos ves que los minutos pasan, y la señora se pone a llorar y es como un drama que además no estamos preparados para nada, ni siquiera como humanos, que vos salís con una angustia de ahí terrible (I1).

FG2 Capaz que sos muy solvente y muy sólido en tu materia, capaz que tenés un muy buen vínculo con los gurises, pero es eso, yo no puedo ni debo enfrentarme a una chiquilina con un problema de depresión, por ejemplo. Y se da cada vez más. O con problemas de aprendizaje. Yo no tengo herramientas. Entonces para mí es eso, venimos tan rezagados, tan rezagados, en la formación del liceo, que sabemos que el sistema educativo de secundaria, para mí es obsoleto, yo no creo... yo estoy dando clase y no creo en lo que estoy haciendo, porque me parece una truchada lo que estoy haciendo (T2).

Day (2012) establece que muchos docentes cuando inician su carrera están convencidos de que su tarea es significativa a nivel social por los cambios que pueda llegar a conseguir. Sin embargo, esta sensación puede variar cuando el docente experimenta una sensación de incoherencia para con su tarea, tal como lo están expresando ambos participantes. Los temas asociados a la formación docente también aparecen en ambos FG con gran frecuencia e intensidad, pero también notamos diferencias significativas entre los dos. Mientras que en el FG1 se hace hincapié en que los docentes no deberían estar formados específicamente para trabajar con las familias o con las dificultades severas con las que se enfrentan en el día a día (sin dejar de valorar como pobre a la formación del I.P.A.), en el FG2 se es muy crítico con la formación de base que reciben los docentes, estableciendo que esta debería ser reformulada en su totalidad.

FG1: No me doy mucha cuenta qué es lo que querría yo en la formación para intervenir. Me parece que escapa un poco a la formación. Me parece que deberíamos tener más equipo, mayor equipo, y no solo en cantidad, en calidad y desde otro lugar, psicóloga, asistente social, psicopedagoga. Yo creo que va más para ese lado, sí (R1).

FG2: Es que es como vos decís. Hay una formación paralela. Está la formación del IPA, y después toda la formación... a mí, por ejemplo, me pasó que yo iba haciendo el IPA e iba haciendo didáctica al mismo tiempo, iba haciendo la formación paralela de la experiencia, y en didáctica obviamente me pasaba eso, y a todos mis compañeros porque todos dábamos clase, nos pasaba que claro, en el deber ser les decías a los profesores de didáctica, claro, tiene razón, por supuesto que vamos a hacer eso sí... y cuando se iba decías, pero esto que dice es imposible, ¿de qué está hablando? (F2). 
En estos pasajes de los FG queda establecido que existe disconformidad para con la formación, aunque las reivindicaciones son distintas. Por un lado, tenemos una docente con más de veinte años de experiencia que solicita apoyo de otros técnicos para trabajar con las familias y no considera necesario continuar formándose en esta área más allá de lo que ya se formó, incluso cuando en el FG se establece en más de una oportunidad que las familias han cambiado y que presentan mayores complejidades. Por el otro lado una docente con cinco años de experiencia hace hincapié en que la formación de base ya es deficitaria de por sí, no es realista y existe un notorio descreimiento en los docentes encargados de llevar adelante la parte práctica de dicha formación.

En la bibliografía sobre formación docente utilizada para este estudio estas dos dimensiones son percibidas y trabajadas. La formación de base es débil (Marrero, 2011; Gimeno Sacristán, 1997; Vaillant 2009; Vaillant, 2010; Marcelo y Vaillant, 2009) y esto evidentemente supone una dificultad a los docentes en el desarrollo de sus tareas tal como es descrito aquí. Por otro lado, está el tema de la formación continua o permanente, que supone una actualización en los saberes de los docentes, quienes deben adaptarse a las transformaciones sociales que acontecen con el paso del tiempo y que se traducen en situaciones conflictivas dentro del aula (Perrenoud, 2010; Marrero, 2011; Gimeno Sacristán, 1997; Vaillant, 2009; Vaillant, 2010).

Se observa en el FG1 una mayor tendencia a reflexionar acerca del rol docente y las acciones que estos realizan en sus trabajos día a día, con muchas de las cuales no están de acuerdo; por otro lado, en el FG2 la tendencia es colocar las responsabilidades por fuera: en las direcciones, en la formación, en las inspecciones, en los programas y en las familias que no se hacen cargo de sus hijos.

Es interesante como un participante del FG2, un docente con 5 años de experiencia y con formación de posgrado, trae el tema de la desactualización de conocimientos que existe por parte de los encargados de formar a los futuros docentes, quienes tienen muchos años de experiencia, pero a su vez se encuentran hace años alejados de las tareas de docencia directa en secundaria

FG2: Pero también tenés que tomar en cuenta que los docentes del IPA, en el caso de los que se dedican a dar didáctica, que se supone que te enseñan a ser docente, eh... hace años que no dan clase. Entonces decís, no... pero lo que vos me estás diciendo no tiene sentido, porque... y te empiezan a mirar mal, porque no, acá lo dice, yo que sé, Vigotsky, y vos te das cuenta de que la ZDP... Pará flaco... (N2).

Es interesante como esta observación surge de un participante que, si bien tiene pocos años de experiencia como docente, acorde con su edad, sí cuenta con una formación de maestría en Historia social y cultural. Perrenoud (2010) establece que entra dentro de las competencias de los docentes el poder gestionar su propia formación continua, lo cual contrasta con una postura de cierta pasividad que se observa en los discursos 
de los docentes, pero no en el caso de este. Al mismo tiempo se perciben en su discurso sus perspectivas acera de cómo el factor edad, experiencia e inmovilidad en los ámbitos de trabajo determina el accionar de los profesionales, lo cual va en la misma dirección que los planteamientos de Day (2005) acerca de las fases del desarrollo de la profesión docente.

Otra de las diferencias que llaman la atención entre ambos FG tiene que ver con la condición de madre o padre y cómo esto puede intervenir en las perspectivas de los docentes para el trabajo con las familias. Rodrigo y Palacios (1998) establecen que el hecho de convertirse en madre o padre constituye una de las transiciones normativas más importantes en la vida de una persona y que esto trae aparejados cambios, tanto en lo emocional como en lo cognitivo.

También es interesante para pensar en este punto el planteo de Day (2005) acerca de las fases del desarrollo profesional de los docentes y cómo se relaciona el interés y entusiasmo de estos por el desarrollo de su profesión con el hecho de convertirse en padres. Tomando en cuenta la etapa de la vida y del desarrollo profesional en la que se encuentra cada docente, las variables relacionadas a su entorno familiar tienen un peso importante (Day, 2012). Por lo tanto, la paternidad es un factor fundamental a tener en cuenta, pero esta tendrá un impacto diferente de acuerdo al momento de su vida profesional en el que se encuentre cada docente.

En el FG1 cuatro de las seis participantes son madres y esto está directamente vinculado con temas importantes que surgen en la discusión: por un lado, una de las participantes establece que a la hora de intervenir en situaciones conflictivas el hecho de ser madre es más importante que el de ser docente.

FG1: Yo a veces les contesto no desde mi rol docente, sino desde el rol de madre. Yo soy más mamá ahí. Contesto más como mamá que como docente. (R1).

Por otro lado, una participante expresa que, a la hora de pensar en el compromiso docente, el tiempo y las energías de las que se dispone están vinculados con la condición de ser madre.

FG1: Es a eso a lo que yo voy cuando te digo lo de las reuniones. Es muy difícil. Yo reconozco, yo no soy madre, ponele que tengo otros tiempos, pero somos muy pocos los docentes que realmente vamos a las reuniones. Cuando hay reuniones, somos las mismas 6 personas que organizamos las quermeses, los campamentos (C1).

En el FG2 solo una de las participantes es madre. En este grupo el tema de la maternidad aparece de un modo distinto, asociado a una postura que presentan las maestras de educación primaria. Ya se había hecho referencia anteriormente a que la 
percepción de desvalorización del rol docente que aparece en este grupo en más de un momento surge en contraposición al rol de la maestra.

Uno de los participantes de este grupo, en relación a esta temática, establece lo siguiente.

FG2: O sea, si tendrá peso la maestra en la formación, que, en realidad, sí, la maestra me mintió, pero no importa, porque me lo dijo la maestra. Y la maestra se lo cree. Eso también es interesante. La maestra se cree el rol de ser una madre sustituta (N2).

Mientras que en el FG2 el tema de la desvalorización de la figura del docente es traído de modo muy cercano a la comparación con la figura de la maestra, vemos que en el FG1 este tema aparece de modo distinto, considerando que la figura del docente tiene su peso y es importante para los alumnos, quienes confían en ellos.

FG1: Es que yo muchas veces les digo, porque ellos buscan al docente.

El docente todavía tiene esa figura muy importante para el niño chico.

Ellos te buscan, ellos te cuentan muchas cosas de ellos (R1).

\section{DISCUSIÓN Y CONCLUSIONES}

Con respecto al primero de los objetivos -indagar acerca de las perspectivas de los docentes de secundaria sobre la importancia de la participación de la familia en la educación-podemos decir que, desde la perspectiva de los docentes, existe acuerdo en que el trabajo con las familias desde las instituciones de educación secundaria y la participación de estas constituyen un factor muy importante y necesario para obtener buenos resultados en los procesos de enseñanza-aprendizaje, así como para resolver otro tipo de situaciones conflictivas que puedan presentar los alumnos, ocupando estas últimas la mayor parte del tiempo y de la dedicación de los docentes.

En este punto se corrobora, de algún modo, lo que aparece en la bibliografía consultada sobre la temática, la que, si bien en su enorme mayoría apunta a la enseñanza primaria, establece la importancia de la participación de la familia como un factor decisivo a la hora de obtener buenos resultados. Con respecto a esto encontramos en las perspectivas de los docentes determinadas dificultades que resultan en que el trabajo con las familias no se desarrolle de la mejor manera. Entre estas aparecen, en cuanto a las familias, la complejidad que presentan la mayoría de las familias de los alumnos con los que trabajan más allá del contexto del que se trate y la ausencia de muchas de las familias que, más allá del modo en que se las convoque, no se acercan a la institución.

De parte de los docentes, la carencia de formación con la que cuentan para hacerse cargo de tareas relacionadas al trabajo con familias y la falta de apoyo desde la institución, ya sea por la inexistencia de equipos multidisciplinarios como por ausencia de lineamientos claros y políticas específicas para dicho trabajo. Los 
docentes señalan, además, la resistencia que existe desde algunas direcciones, así como también de algunos docentes para acercar a las familias a la institución. Existen por otro lado dificultades para emprender acciones desde lo colectivo, con el objetivo de mejorar esta situación y se destacan los escollos a nivel reglamentario y burocrático que existen para que la familia participe en la institución educativa en secundaria. Aparece subrayada la dificultad que se le plantea a los docentes a la hora de definir su rol profesional el tener que trabajar con las familias de los alumnos.

En lo que respecta al segundo objetivo -indagar acerca de qué diferencias entienden los docentes que existen en la participación de la familia en la educación entre los sistemas de primaria y secundaria- encontramos que los docentes señalan que la participación de la familia tiene lugar de modos muy distintos entre los sistemas de primaria y secundaria, tendiendo en este último, a disminuir la participación de modo abrupto. En la bibliografía consultada para este estudio, esta temática es nombrada la enorme mayoría de las veces como la relación familia-escuela (Comellas, 2009; Dabas, 1998; González, Vandemeulebroecke y Colpin, 2001), dejando establecido, más allá de los modos en que se nombre a la institución educativa en cada contexto particular, que la participación es generalmente mucho mayor en las instituciones de educación primaria. Los docentes expresan que esta situación se da porque existen creencias en los padres, así como en otros actores institucionales, que marcan que el niñoadolescente, una vez que obtiene el pasaje a secundaria, ya no necesita de la participación de la familia. Por su lado, las instituciones de educación secundaria no tienen entre sus objetivos el acercar a las familias, y en muchas instituciones existe una falta total de lineamientos para el trabajo con estas, así como carencia de respuestas ante las situaciones conflictivas que se presentan en el día a día. Varios actores involucrados en la educación secundaria, como direcciones y docentes, tienen resistencias a la hora de convocar a las familias, y existen mecanismos burocráticos que impiden a estas participar en educación secundaria tal como lo hacían en primaria. Según estos docentes, en el colectivo social existe un respeto, cuidado y valoración de la escuela que no se repite en las instituciones de educación secundaria. Para muchas familias los aprendizajes que se obtienen en educación secundaria no son importantes; la figura de los docentes de secundaria está desvalorizada y es percibida de este modo también por las familias. Por último, en lo que respecta al tercer objetivo - conocer sobre la formación con la que cuentan los docentes de secundaria para el trabajo en la relación familia-institución educativa - encontramos que los docentes de educación secundaria no cuentan en su formación de base con ningún tipo de preparación para el trabajo con las familias. De los docentes con los que se trabajó en los focus group, dos de ellos contaban con formación de posgrado, y en una de ellas se incluía un seminario destinado al trabajo con familias.

En la bibliografía consultada para este estudio sobre formación docente en secundaria se pudo observar que esta es deficitaria, no se encuentra actualizada y no produce conocimientos. Tal como se hizo referencia anteriormente, en Uruguay se ha 
evaluado a la formación de docentes de secundaria como una de las más débiles dentro de América Latina. Con respecto a esto, los docentes expresan lo siguiente: la formación docente es percibida como pobre, desactualizada, desajustada para con la realidad; tiene una carencia total en lo que respecta al trabajo con las familias, pero esta no es la única. La formación no consigue alcanzar respuestas para el gran número de dificultades que se le presentan a los docentes en sus prácticas cotidianas, como ser el incremento de las matrículas, el incluir alumnos con dificultades de todo tipo y el no contar con los recursos adecuados. Algunos docentes expresan que no deberían formarse para trabajar con las familias ni con todas las dificultades, sino que deberían contar con apoyo de otros técnicos.

Las contradicciones existentes entre la teoría y la práctica que se producen en la formación docente determinan dificultades en los docentes para definir su rol. Por otro lado, la falta de herramientas con las que cuentan los docentes para desarrollar su tarea genera descreimiento y hasta desvalorización de la tarea que están realizando. La formación que obtienen los docentes por su propia experiencia es generalmente más valiosa que la obtenida en los institutos. Según estos docentes, en las situaciones conflictivas es más importante el factor afectivo y el componente vocacional con el que cuentan que las herramientas que les ofrece su formación. Consideramos que a lo largo del trabajo las preguntas que se formularon han encontrado respuestas, que hemos repasado junto con los objetivos trazados al comienzo del estudio.

\section{REFERENCIAS}

Alfiz, I. (1997). El proyecto educativo institucional. Propuestas para un diseño colectivo. Buenos Aires, Argentina: Aique.

Bardin, L. (1986). Análisis de contenido. Madrid, España: Akal.

Castro, J.,y Regattieri, M. (2012).Interacción escuela familia. Insumos para las prácticas escolares. Brasilia, Brasil: UNESCO - MEC.

Comellas, M.J. (2009). Familia y escuela: compartir la educación. Barcelona, España: Grao.

Coulon, A. (1995). Etnometodología y educación. Buenos Aires, Argentina: Paidós.

Dabas, E. (1998).Redes sociales, familias y escuelas. Buenos Aires, Argentina: Paidós.

Day, C. (2005).Formar docentes. Cómo, cuándo y en qué condiciones aprende el profesorado. Madrid, España: Narcea.

Day, C. (2012).Pasión por enseñar. La identidad personal y profesional del docente y sus valores. Madrid, España: Narcea.

Flick, U. (2004).Introducción a la investigación cualitativa. Madrid, España: Morata.

Gallo, P. (2009). Transformaciones en las relaciones intergeneracionales, Autoridad y Violencia en las escuelas. En G. Noel(Comp.), La violencia en las escuelas desde una perspectiva cualitativa (pp. 7-20). Buenos Aires, Argentina: Ministerio de Educación de la Nación. 
Gimeno Sacristán, J. (1997).Docencia y cultura escolar. Reformas y modelo educativo. Buenos Aires, Argentina: Lugar editorial.

Glaser, B., y Strauss, A. (1967). The discovery of grounded theory: strategies for qualitative research. New York, NY: Aldine Publishing Company.

González, M., Vandemeulebroecke, L., y Colpin, H. (2001). Pedagogía familiar. Aportes desde la teoría y la investigación. Montevideo, Uruguay: Trilce.

González Tornaría, M.L., y Wagner, A. (2011).La relación familia-escuela: perspectivas y formación de docentes brasileros y uruguayos (Proyecto de investigación). Universidad de la República/UniversidadCatólicadelUruguay/UniversidadFederaldeRioGrandedoSul.

Lacasa, P. (1997).Familias y escuelas. Caminos de la orientación educativa. Madrid, España: Visor.

Ley № 18.437. (12 de diciembre de 2008). Ley General de Educación. Recuperado de http://www.impo.com.uy/bases/leyes/18437-2008

Marcelo, C., y Vaillant, D. (2009).Desarrollo profesional docente. Madrid, España: Narcea.

Marrero, A. (2011).La formación docente en su laberinto. Los debates, los actores y una ley. Montevideo, Uruguay: Ediciones Cruz del Sur.

Maxwell, J. (1996). Qualitative research design. An interactive approach. Thousand Oaks, CA: Sage.

Minuchin, S., y Fishman, H. (1984). Técnicas de terapia familiar. Barcelona, España: Paidós.

Palacios, J., y Oliva, A. (2008). La adolescencia y su significado evolutivo. En J. Palacios, A. Marchesi, y C. Coll(Comps.), Desarrollo psicológico y educación. 1. Psicología evolutiva (p. 433-451). Madrid, España: Alianza.

Paredes, M. (2003). Los cambios en la familia en Uruguay: ¿Hacia una segunda transición demográfica? En Nuevas formas de familia. Perspectivas nacionales e internacionales (p. 73-102). Montevideo, Uruguay: UNICEF - UDELAR.

Pérez de León, P. (2001). Focus group. Sistematización de prácticas y calidad. Prisma, 16, 165-179.

Perrenoud, P. (2010).Diez nuevas competencias para enseñar. Barcelona, España: Grao.

Rodrigo, M.J., y Palacios, J. (1998).Familia y Desarrollo humano. Madrid, España: Alianza.

Rodrigo, M.J., Máiquez, M., Martín, J., y Byrne, S. (2008).Preservación familiar. Un enfoque positivo para la intervención con las familias. Madrid, España: Pirámide.

Vaillant, D. (2009). Formación de profesores de Educación Secundaria: Realidades y discursos. Revista de Educación, 350, 105-122.

Vaillant, D. (2010). Capacidades docentes para la educación del mañana. Pensamiento iberoamericano, 7, 113-128.

Vasilachis de Gialdino, I. (Coord.). (2007).Estrategias de investigación cualitativa. Buenos Aires, Argentina: Gedisa.

Walsh, F. (2004).Resiliencia Familiar: estrategias para su fortalecimiento. Buenos Aires, Argentina: Amorrortu. 